\title{
Lipopolysaccharide, structure and biological effects
}

\author{
Clyde Schultz* \\ Department of Biology, The University of Calgary, Calgary, Alberta, Canada T2N 1N4
}

\begin{abstract}
Lipopolysaccharide is an integral part of the cell wall of Gram negative bacteria. It is a complex molecule that is involved in a variety of functions both related to the cell to which it is comprised and the extracellular environment. It is composed of three regions, with two of the three exposed to the external environment. Lipopolysaccharide is serologically active in that host specific antibodies are produced that react with the O-side chains which comprise outer most region of the lipopolysaccharide structure. The endotoxic component resides in Region III which can cause fatal sepsis in high enough concentrations.
\end{abstract}

\section{Introduction}

Lipopolysaccharide (LPS) is a molecule that comprises part of the bacterial cell wall of Gram negative bacteria and assists in stabilizing the bacterial cell wall. Gram negative bacteria are the only organisms in nature which possess LPS and Lipid A which is the "endotoxic" component. The molecular weight and structure will vary with the species of bacteria, as will the actual pathogenicity of the LPS itself. LPS is composed of three parts. The $\mathrm{O}$-side chain, a middle polysaccharide component, and Lipid A which is the actual pyrogenic component of LPS. Lipid A is embedded in the cell wall of Gram negative bacteria. The other two components face outward, away from the bacterial cell wall. Lipid A serves as an anchor for the LPS component. There are other substances that may produce pyrogenic (fever causing) responses such as plastics, but the potentially life-threatening substance is Lipid A from LPS.

\section{Structure}

LPS serves as an internal, and external part of the outer cell wall and inner membrane of Gram negative bacterial cells [1,2]. It provides a stabilizing and protecting structural support to the cell and helps to insure integrity of the cell wall and thus survival of the cell especially during conditions of extreme stress, such as desiccation [1,2]. LPS is composed of three regions which vary in homogeneity, structure and specific location. It is the outermost part of the molecule with substructures that may be completely exposed to the extracellular space yet is anchored by Region 3 which is not exposed at all to the outside of the cell.

The O-specific polysaccharide region (Region 1) is the primary site of immunologic specificity. It is in general a repetitive glycan polymer. The $\mathrm{O}$ antigen may contain 100 or more oligosaccharide units composed of 3 to 4 monosaccharides. The various monosaccharides may contain pentoses, hexoses and de-oxy sugars, and uronic acids, with amino groups being $\mathrm{N}$-acetylated [2]. It is, in other words heterogeneous. This region is variable both within species and between species and is attached to region II. It is completely exposed to the extracellular environment [3]. Biosynthesis occurs in 4 steps. First is the initial assembly of $\mathrm{O}$ antigen repeating units. These are lipid intermediates and are comprised of sugar phosphates and a phospho carrier lipid. Step 2 is the polymerization of these units. All of which are attached to membrane bound PP lipid [4]. Thirdly is the enzymatic transfer of these formed O polymers to the LPS core polysaccharide (Region II). Lastly is the regeneration of the carrier lipid (P-carrier or monophosphopolyisoprenol.

The O-side chain is also used to make vaccine, especially for protection against infectious organism. Immunity is often long lasting as T memory cells are produced. As such, it is specific for a given microbial serotype $[4,5]$. The resulting protection that comes with the programming of $\mathrm{T}$ memory cells, results in the conference of secondary immunity.

The core polysaccharide component (Region II) is adjacent to the Lipid A substructure and is also exposed to the extracellular environment [2]. It is constructed by membrane associated enzymatic activity that catalyze the addition of monosaccharides to the reducing terminus of the core polysaccharide [5,6]. These come from the metabolism of nucleotide sugar intermediates. The addition sequence of 2-keto-deoxy-octulosonic acid (KDO), heptose, phosphate and ethanolamine are goverened by specific enzyme activity of the acceptor and activated nucleoside sugar donor. KDO binds to Lipid A by a ketosidic linkage. Mutants that cannot synthesize KDO are non-viable [5].

The Lipid A substructure (Region III) of LPS has the threedimensional appearance of common soap. It is the most structurally conserved part of the LPS structure. It has long fatty acid side chains (approximately) 16 carbons) and is very durable [3]. Purified Lipid A can withstand extremes in heat and pressure with its biological activity intact. The core of Lipid A is comprised of B-glucosamine(1-6)-glucosamine-1-phospahte base with fatty acid esters attached to the carbohydrates [4]. It is synthesized by addition of fatty acids and KDO to glucosamine disaccharides. The associated acyl chain groups are largely conserved within a bacterial species and are synthesized in a series of five steps initiated by an acylation step and culminating with a KDO construct that acts as the anchor for the rest of the molecule.

Correspondence to: Clyde Schultz, University of Calgary, Alberta, Canada T2N 1N4, Tel: 403.220.5278; E-mail: schultzc@ucalgary.ca

Key words: Endotoxin, Pyrogen, Lipid A, polysaccharide, fever

Received: January 03, 2018; Accepted: January 21, 2018; Published: January 24 2018 


\section{Biological effects and pathogenicity}

Pathogenicity due to the LPS presence is almost entirely due to the Region III component, Lipid A, which in the older literature is referred to as catechin. Overt pathogenicity of Lipid A may initially manifest itself by the development of fever and other changes in metabolism in small doses [4,7]. Body temperature (fever) may rise quickly in individuals infected with lysed Gram-negative bacteria [8]. As Gramnegative bacteria are lysed by the cellular network, the cell walls are broken apart releasing Lipid A to bind with other effector cells such as macrophages. Lethal doses may can result in septic shock, hypotension, in addition to fever. Lipid A acts to signal macrophages to produce pyrogens which in turn with activate mediators such as prostaglandins $[7,8,9]$. Prostaglandins act directly on the temperature controls in the brain. The development of symptoms largely depends on the animal species, route of inoculation, dose and timing of systemic release. Binding begins the inflammatory cascade, causing cells to produce and release a variety of cytokines, including IL-1, IL-6, and TNF [7]. The activated cytokine network has multiple and often overlapping effects, including but not limited to complement activation $[10,11]$.

Part of the pathogenicity of Lipid A comes from its' ability to activate the immune system. When Lipid A is detected by cells in the blood cytokines such as TNF and Interleukin 2 are activated [11]. These molecules have a variety of functions including cell activation, complement activation, and stimulation of the release of other cytokines, such as IL-6. These molecules can also be tissue destructive on their own. The cytokines are a major "communication" network among cells and may act very rapidly, especially related to allergic reactions [12].

\section{Summary}

LPS is varied and important biological molecule for a variety of reasons, chiefly to human health. The Lipid A component can cause death or shock at the appropriate levels. As such it is important to be able to detect the it not only in the human body but also in the pharmaceutical industry. There are various well published and sensitive techniques for detecting endotoxin in liquids and on medical devices. The major issue with some of these techniques is that they will detect potentially any pyrogen, not just LPS. These organic molecules are often mistakenly referred to as "endotoxin" and may cause a pyrogenic response but are not the integral part of the LPS molecule that Lipid A is part of. Distinctions and an understanding of this fundamental and yet real difference is key to both patient care and pharmaceutical manufacturing.

\section{References}

1. Rietschel E (1994) Bacterial Endotoxin: molecular relationships of structure to activity and function. FASEB J 8: 217-225.

2. Raetz C (2000) Lipopolysaccharide Endotoxins. Annu Rev Biochem 71: 635-670 [Crossref]

3. Warren H (2000) Resilience to bacterial infection: differences between species could be due to proteins in serum. J Infect Dis 201: 223-232.

4. Emiola A (2015) A complete pathway model for Lipid A Biosynthesis in E. Coli. PLoS One 10: e0121216. [Crossref]

5. Hershberger C (1968) Chemistry and metabolism of 3-deoxy-D-mannooctulosonic acid. I. Stereochemical determination. J Biol Chem 243: 1578-1584.

6. Walker S (2004) Role of cell surface lipopolysaccharide in Escherichia coli K12 dhesion and transport. Langmuir 20: 7736-7746. [Crossref]

7. Moran A (1996) Molecular mimicry of host structures by bacterial lipopolysaccharides and its contribution to disease. FEMS Immunol Med Microbiol 16: 105-115.

8. Kilar A (2013) Structural characterization of bacterial lipopolysaccharides with mas spectrometry and on-and off-line separation techniques. Mass Spectrom Rev 32: 90117.

9. Reeves P (2002) Genomic organization of LPS-specific loci. Curr Top Microbial Immunol 264: 109-135. [Crossref]

10. Walport M (2001) Complement. First of two parts. N Eng J Med 344: 1058-1066. [Crossref]

11. Ricklin D (2010) Complement-a key system for immune surveillance and homeostasis Nat Immunol 11: 785-797. [Crossref]

12. Williams L (2005) The role of endotoxin and its receptors in allergic disease. Ann Allergy Asthma Immunol 94: 323-332. [Crossref]

Copyright: (C2018 Schultz C. This is an open-access article distributed under the terms of the Creative Commons Attribution License, which permits unrestricted use, distribution, and reproduction in any medium, provided the original author and source are credited. 